\title{
Giant Frontal Osteoma: Case Report with Review of Literature
}

\author{
Rajesh Vishwakarma $\cdot$ Shawn T. Joseph $\cdot$ \\ Kalpesh B. Patel • Arpit Sharma
}

Received: 23 March 2008/Accepted: 21 August 2008/Published online: 20 April 2011

(C) Association of Otolaryngologists of India 2011

\begin{abstract}
Diagnosis of frontal osteoma is usually by chance, but rarely these can produce exceptional ophthalmologic and neurological complications apart from cosmetic disfigurement. Etiology of frontal osteoma may be multifactorial. Surgical management should be site and size specific. A combination of open surgery and endoscopic methods would help confirm complete removal of the tumor. Here we report a fronto-ethmoidal osteoma of size $7.1 \times 5.3 \times 5.1 \mathrm{~cm}$ which is one of the largest reported in literature. Also, we have done an extensive web search and text based review of the literature on frontal osteoma in terms of its incidence, etiology, pathology, clinical presentation, complications and important developments in management. The available literature and our own experience suggest that even large osteoma arising in the fronto-ethmoid region can be completely removed surgically with minimum complications. The surgical approach can be varied according to the extent of the tumor and patient considerations. A regular follow up is necessary in asymptomatic cases being treated conservatively, in view of the potential complications.
\end{abstract}

Keywords Osteoma - Frontal sinus - Proptosis · LynchHowarth approach · Headache $\cdot$ Endonasal surgery ·

Sinusitis

\section{Introduction}

Skull base osteomas are rare neoformations which often remain asymptomatic throughout a patient's life. Diagnosis

R. Vishwakarma · S. T. Joseph $(\bowtie) \cdot$ K. B. Patel · A. Sharma Government Medical College, Bhavnagar, Gujarat, India e-mail: shawndr@gmail.com is usually by chance, but rarely these can produce exceptional ophthalmologic and neurological complications apart from cosmetic disfigurement. An in depth understanding of this tumor and its complications thus becomes important. Here we have carried out an extensive internet and text based review covering the incidence, etiology, pathology, clinical features, complications, diagnosis and treatment of frontal osteoma and summarized the important observations. We report a giant frontal osteoma which is one of the largest ever reported in literature. This multicompartmental osteoma was removed en bloc by a single stage surgery. A combination of open surgery and endoscopic methods could help confirm complete removal of the tumor.

\section{Giant Frontal Osteoma: Case Report}

A 20 year old male presented to us with complaints of frontal headache, recurrent upper respiratory tract infections of 2 months duration and protrusion of left eye ball noticed 15 days back. There was no history of trauma or significant medical events in the past. Examination revealed left sided proptosis with inferolateral displacement, vertical diplopia and evidence of frontoethmoidal sinusitis. Fundoscopy was normal. CECT Brain and orbit showed a giant osteoma of the left frontal and ethmoid sinuses, expanding them and projecting into the superomedial aspect of left orbit causing proptosis and retained secretions in right frontal sinus. The lesion was bulging into the left subfrontal region focally lifting dura and compressing brain. After appropriate pre operative preparations surgery was performed by a fronto orbital approach using a Lynch-Howarth incision. A giant osteoma was seen involving the left frontal and ethmoid sinus pushing 


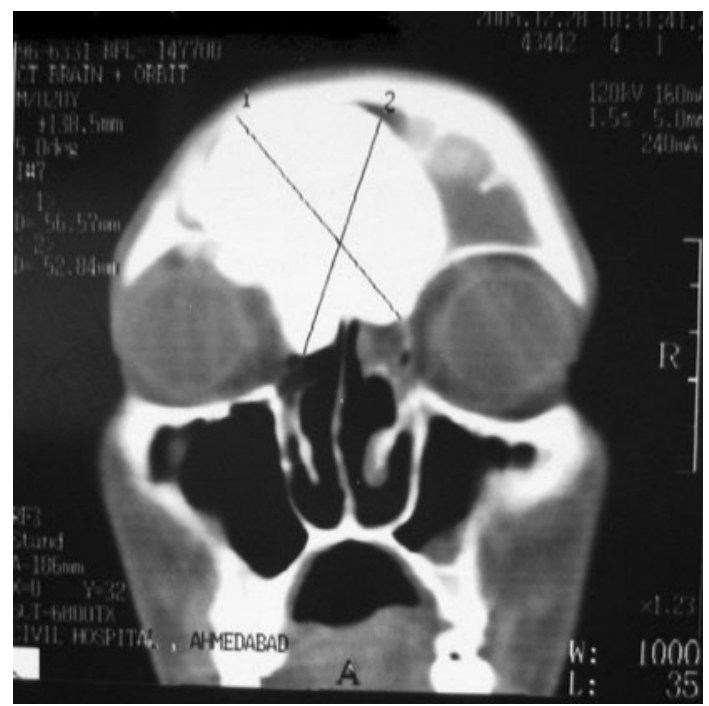

Fig. 1 Contrast enhanced CT scan showing the radiological dimensions of the osteoma

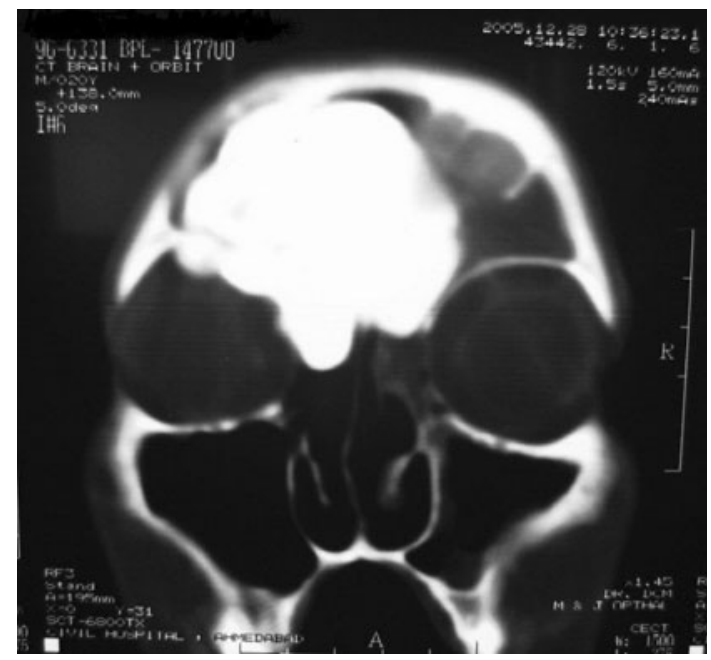

Fig. 2 CT scan showing the orbit being pushed inferolaterally

the orbit inferolaterally, frontal lobe of brain posteriorly and interfrontal septum to the right side. Polyps were seen in the frontal and ethmoid sinuses. The tumour was removed en bloc, polypectomy done along with frontoethmoidal clearance. The tumour measured $7.1 \times 5.3 \times$ $5.1 \mathrm{~cm}$ which is one of the largest reported in literature. No sinus obliteration was performed. Post operatively the patient had cerebrospinal fluid rhinorrhoea, which was completely cured by conservative treatment. (Fig. 1), (Fig. 2), (Fig. 3), (Fig. 4), (Fig. 5) (Fig. 6).

\section{Discussion}

Diagnosis of frontal osteoma is usually by chance, but rarely these can produce exceptional ophthalmologic and

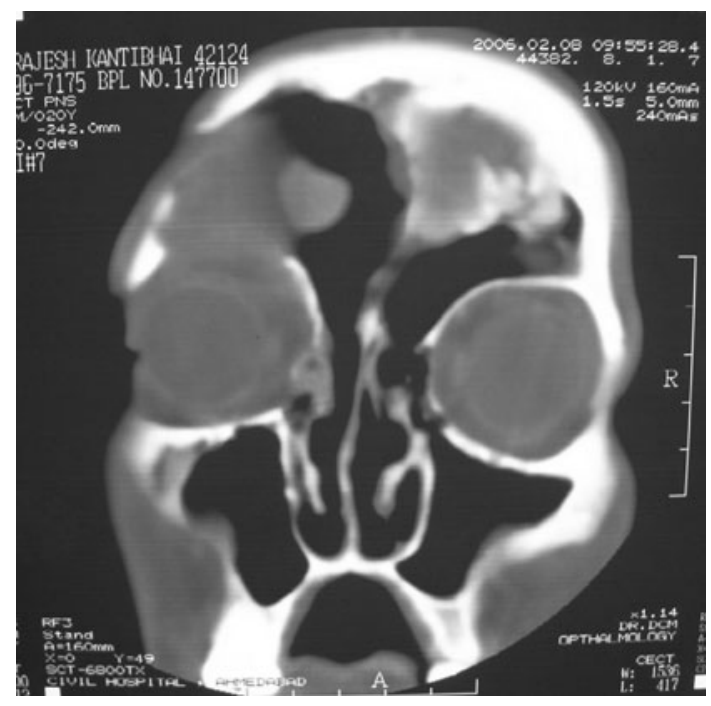

Fig. 3 Post operative CT scan of the same patient after excising the osteoma

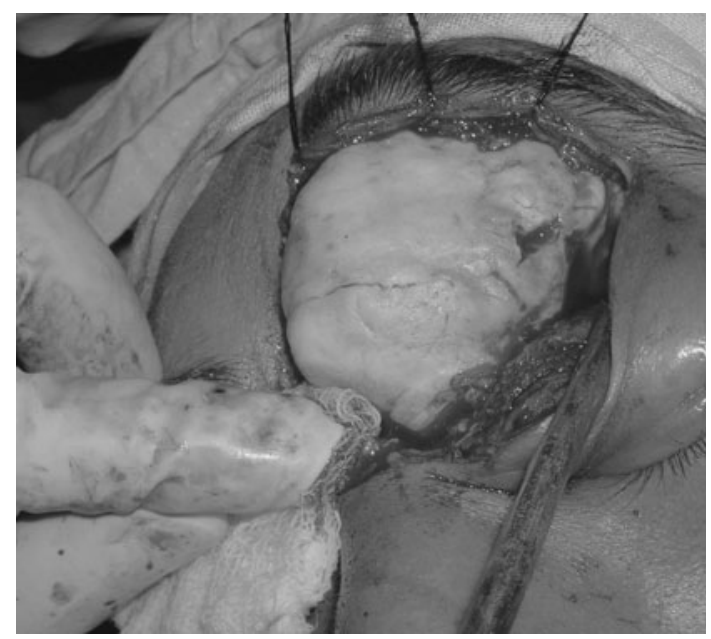

Fig. 4 Frontal osteoma being removed by Lynch-Howarth approach

neurological complications apart from cosmetic disfigurement. Most common presentation is headache though sinusitis and forehead deformity are not infrequent [1]. None of the present theories fully explain the etiopathogenesis of frontal osteoma. A multifactorial etiology should be strong possibility. Surgical management should be site and size specific. Purely endoscopic method may be used for small osteomas of fronto ethmoid localization or the anterior wall of frontal near the midline. The risk of incomplete removal with this approach can be reduced by image guided surgery. Open surgery should be the method of choice in larger and complicated frontal osteomas. A combination of open surgery and endoscopic methods would help confirm complete removal of the tumor in large frontal osteoma. Complete surgical excision is curative. 


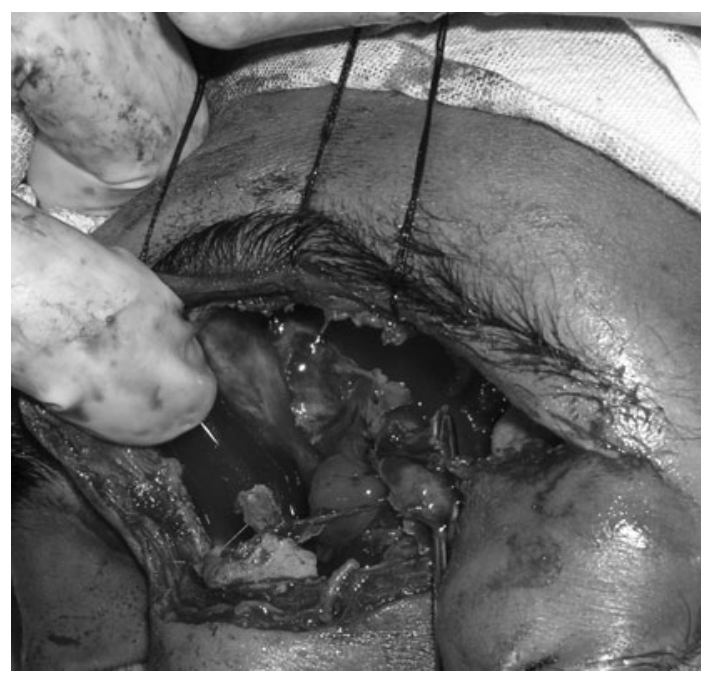

Fig. 5 Mucocoele in frontal sinus seen after removal of the osteoma. The compression of brain matter can also be seen

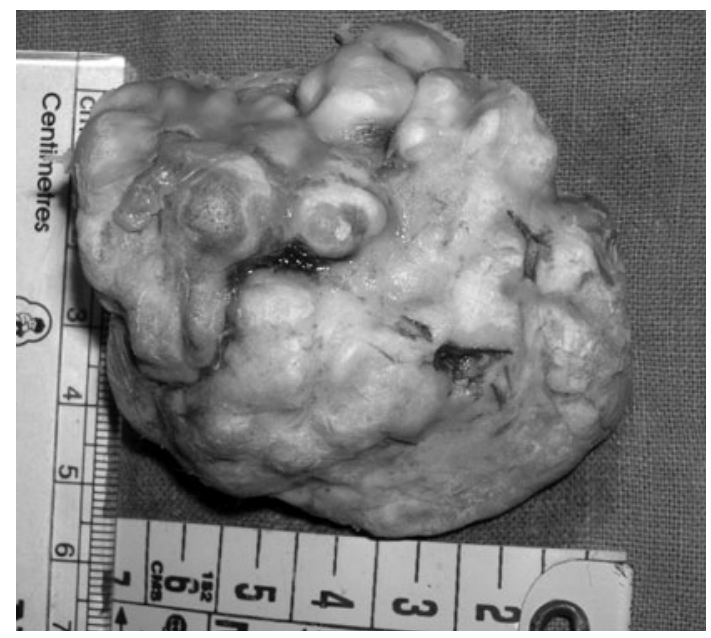

Fig. 6 Clinical photograph of the excised osteoma showing dimensions

\section{Incidence}

The accurate incidence of this tumour is difficult to elucidate since most of the cases are asymptomatic and present late. Referral pattern bias adds to this. Nevertheless, a fairly good assessment can be reached if one studies the available information. Osteomas constitute $1 \%$ of all bone tumors and $11 \%$ of benign bone lesions [2] It is usually seen in the second and third decades of life $(75 \%)$ [3] with a male predominance [3] of 2:1. In our series of patients $71.43 \%$ of patients were in the second and third decades of life and male to female ratio was 5:2. Cranial osteomas generally arise from the skull vault. Skull base osteomas are rare and occur in the frontal and ethmoidal sinuses. Very rarely these can occur in the maxillary and sphenoid sinuses. 47 to $80 \%$ of paranasal sinus osteomas are found in the frontal sinus, $37 \%$ of these originating near the frontal sinus ostium and the others from the roof, floor, interfrontal septum, anterior or posterior wall. In the frontoethmoidal localization it is most commonly located near the nasolacrimal duct.

\section{Aetiology}

Three theories have been put forward regarding the etiopathogenesis of paranasal sinus osteoma [4, 5]. These include traumatic, infective and embryological [4-6]. A history of trauma may precede the development of an osteoma. It has been observed that trauma during puberty, when the bone development is maximum, may be associated with osteoma. Sinus infection and inflammation has been proposed in the etiology of osteoma. Sinusitis seems more likely to be a secondary event than a primary event in majority of cases. Embryological theory says that an osteoma would originate in the fronto-ethmoidal sutures where the membranous and cartilaginous tissues meet during embryonic life. This seems to be a more acceptable theory but the precipitating event may be recurrent infection or trauma.

\section{Pathology}

Osteomas are bosselated, round to oval sessile tumors that project from the subperiosteal or endosteal surfaces of the bony cortex. Histologically, osteomas are composed of a composite of woven and lamellar bone that is frequently deposited in a cortical pattern with haversian like systems. Some variants contain a component of trabecular bone in which the intertrabecular spaces are filled with haematopoeitic marrow. Thus three types of osteoma can be distinguished $[4,7]$.

- eburnated, compact or ivory, with bony lamellae arranged in parallel layers, slow growing;

- spongy, areal-looking, with wide medullary spaces, without Haversian' channels, of more rapid and more aggressive growth;

- mixed, in which two of the characteristics described above are present and representing the most common form $(>50 \%)$.

\section{Clinical Presentation}

Clinically, most of the frontal sinus osteomas are asymptomatic. Some osteomas become symptomatic in the second to fifth decades of life with a male to female ratio of $2: 1$. This 
may be attributed to the greater exposure of the male gender to trauma and to the larger size of their sinuses. Also the mean growth rate is $1.61 \mathrm{~mm} /$ year, range of 0.44 to $6.0 \mathrm{~mm} /$ year [7]. The most frequent symptom is headache which is characteristically nocturnal and relieved by acetyl salicylic acid. This can also be related to the size and localization of the osteoma but need not be so always. There have been cases of frontal osteoma presenting with epistaxis as a secondary complication. There can be a deformity of the forehead related to the site and size of the osteoma [6, 8]. Complications can be ophthalmological, sinus related or intracranial. Ophthalmological complications are rare and consist of proptosis, diplopia, ptosis $[6,8]$. Very rarely visual loss and epiphora can occur due to compression of lacrimal sac by the osteoma. A few cases of palpebral abscess secondary to frontal osteoma have been reported in literature [7]. Sinus related complications of frontal osteoma include frontal mucocoele, frontal sinusitis and the vacuum 'sinus' syndrome. It has been reported in literature that meningitis [9], cerebral abscess [4, 7, 9], pneumocephalus [10], compression of brain and endocranial hypertension [7,9] can occur due to extension of osteoma into the cranial fossa through the posterior wall of the frontal sinus or the cribriform plate. George $\mathrm{J}$ et al. have reported frontal osteoma in an elderly man, who presented with clinical features of dementia, later found to have an associated intracranial aerocele [11]. Multiple osteomas can be seen associated with polyposis of the large intestine, Gardner's syndrome, subcutaneous desmoid tumors, epidermoid cysts and fibro osseous disease [3, 12].

\section{Diagnosis}

Osteomas are easily demonstrated by plain radiographs [8]. But diagnosis and exact localization of osteoma can be confirmed with the aid of CECT Brain with orbit and paranasal sinuses which shows the extension and the relationship of the osteoma with the adjacent structures. Osteoid osteoma appears as a radio opaque lesion with a nidus which has a radiolucent centre surrounded by dense sclerosis [3]. Nuclear Magnetic Resonance can be useful in cases with suspected endocranial or orbital complications. Spiral CT can be used to give a 3 dimensional reconstruction of the tumor [9]. Radionuclide bone scan can help to differentiate an actively growing lesion (hot) from a stable lesion (cold) [8]. Orbital venography has been used to demonstrate compression of the superior ophthalmic vein.

\section{Treatment}

Asymptomatic osteomas, especially in elderly patients do not require any treatment except a radiographic follow up
[6]. A more aggressive approach may be taken if it shows significant growth or complications. Neuro-ophthalmological and endosinusal complications call for radical surgery. Osteomas producing mechanical complications like ostial obstruction, facial deformity or proptosis are indications for surgery. Those producing a "hot' radionuclide bone scan should also be considered as candidates for surgery. A 'cold' bone scan indicates the presence of a relatively inert osteoma, in terms of biologic growth activity and a nonoperative approach may be adopted if the patient is not symptomatic. The treatment of an uncomplicated osteoma consists of en bloc resection or curettage of the tumor. Selection of surgical approach should depend on the location and extent of the osteomas and any complication, if present. In the orbital region, anterior lesions can be removed via an anterior orbitotomy, while more posterior tumors require an orbitocranial procedure [6]. Mugliston et al. [13] suggest applying, to the surgical treatment of the osteoma, the same evaluative criteria proposed for malignant tumours in the same localizations [7]:

- evaluation of intracranial disease

- protection of the brain

- avoidance of CSF leaks

- adequate haemostasis

- facilitation of resection

The surgical approaches to resection of frontal osteoma are varied. The fronto-orbital approach according to Ogston-Luc is a useful technique in patients with no signs of orbital and/or endocranial compression. The transfacial approach through a Howarth-Lynch incision extended to lateral rhinotomy and the realization of a nasofrontal flap allows good access to the orbital region and to the frontal and posterior ethmoid. This along with endoscopic clearance of sinuses was effective in ensuring complete removal of osteoma as well as treat associated sinus complications. A bicoronal bifrontal ostoplastic technique with the aid of surgical microscope is very useful in large osteomas growing towards the orbit or the endocranium. The bicoronal incision gives a good approach to the frontal sinus, the ethmoid and the eyeball. But this should be avoided in cases of osteomyelitis of the frontal bone and in patients with history of previous frontal sinus surgeries due to the possibility of delayed healing, spread of infection, flap necrosis and possible cosmetic disfigurement. The use of an endocranial approach or mixed craniofacial approach has been recommended by some authors in cases of endocranial complications [8]. Bourgeois $\mathrm{P}$ et al. report the use of a frontobasal craniotomy with complete osteoma and mucocoele removal and cranio facial bone reconstruction in a patient of frontal osteoma with intracranial complications [14]. A combined sinus endoscopic and orbital approach with or without the assistance of stereotactic 
localization can be useful alternative to orbitocranial approach according to some authors [15]. A purely endoscopic endonasal approach can be used for small osteomas of fronto-ethmoid localization and those arising from the anterior wall near midline. This has the risk of incomplete excision but can be reduced by the aid of stereotactic localization. Piece meal excision may be attempted in larger osteomas. Post operative complications include ptosis, diplopia, supraorbital nerve anaesthesia, cerebrospinal fluid rhinorrhoea, most of which respond well to conservative measures [15]. Rappaport JM et al. report the resurfacing of a post operative dural defect using a flap of pericranium to facilitate anterior cranialization [10].

The incidence of recurrence rate after incomplete resection may be up to $10 \%$, according to some authors [2].

Frontal osteomas can be of concern when symptomatic or complicated. Those osteomas presenting early in life, long standing facial asymmetry, proptosis, globe displacement etc., in general, have bad prognosis [12]. A complete surgical resection can be curative. But an incomplete removal can lead to recurrence. Complications can be prevented if the diagnosis is not delayed.

\section{Conclusion}

Frontal osteomas can produce exceptional neurological and ophthalmologic complications. Clinical presentation and complications are site and size specific. Even giant frontal osteomas can be safely removed by careful open surgery. A complete excision is curative. A purely endoscopic endonasal approach has the risk of incomplete excision but can be reduced by the aid of stereotactic localization. Obliteration of the frontal sinus is not mandatory in all cases. Post operative CSF leak, if present, can be managed conservatively, unless too large.

\section{References}

1. Watelet JB, Van Cauwenberge P (1999) Applied anatomy and physiology of the nose and paranasal sinuses. Allergy 54(Supp 57):14-25

2. Grayeli AB, Redondo A, Sterkers O (1980) Anterior skull base osteoid osteoma: case report. Br J Neurosurg 12:173-175

3. Rosenburg A (1999) Bone forming tumours. In: Cotran RS, Kumar V, Collins T (eds) Robbins pathologic basis of disease, 6th edn. Saunders, Philadelphia, p 1235

4. Vowles RH, Bleach NR (1999) Imaging case study of the month: frontoethmoidal osteoma. Ann Otol Rhinol Laryngol 108: $522-524$

5. Gil Tutor E (1998) Osteomas fronto-etmoidales. Ann Otorrinolaringol Ibero Am 25:137-146

6. Selva D, White VA, O’Connnell JX, Rootman J (2004) Primary bone tumours of the orbit. Surv Ophthalmol 49:328-342

7. Dispenza C, Martines R, Dispenza F, Caramanna C, Saraniti C (2004) Frontal sinus osteoma complicated by palpebral abscess: case report. Acta Otorhinolaryngol Ital 24:357-360

8. Mansour AM, Salti H, Uwaydat S, Dakroub R, Bashshour Z (1999) Ethmoid sinus osteoma presenting as epiphora and orbital cellulites: case report and literature review. Surv Ophthalmol 43:413-426

9. Sente M, Topolac R, Peic-Gavran K, Alenksov G (1999) Frontal sinus osteoma as a cause of purulent meningitis. Med Pregl $52: 169-172$

10. Rappaport JM, Attia EL (1994) Pneumocephalus in frontal sinus osteoma: a case report. J Otolaryngol 23(6):430-436

11. George J, Merry GS, Jellett LB, Baker JG (1990) Frontal sinus osteoma with complicating intracranial aerocele. Aust N Z J Surg 60(1):66-68

12. Aylin Y, Murat A, Alpay H, Ramazan A, Bumin D (2004) Orbital extension of a large frontal sinus osteoma associated with fibrous dysplasia. Eur J Gen Med 1(4):74-77

13. Mugliston TAH, Stafford N (1985) A cranio-facial approach to large osteomas of the frontoethmoidal region. J Laryngol Otol 99:979-983

14. Bourgeois P, Fichten A, Louis D, Vincent C, Pertuzon B, Assaker R (2002) Frontal sinus osteomas: neuro-ophthalmological complications. Neurochirurgie 48(2-3 Pt 1):104-108

15. Selva D, Chen C, Wormald PJ (2003) Frontoethmoidal osteoma: a stereotactic-assisted sino-orbital approach. Ophthal Plast Reconstr Surg 19(3):237-238 